\title{
Complicações graves em paciente pediátrico com pneumonia adquirida na comunidade: relato de caso
}

\author{
Severe complications in pediatric patient with community-acquired pneumonia: case report \\ Complicaciones graves en un paciente pediátrico con neumonía adquirida en la \\ comunidad: reporte de caso
}

\begin{abstract}
Matheus Gonçalves de Sousa ${ }^{1 *}$, Edson Barroso dos Santos Júnior ${ }^{1}$, Vitor Toshio Katuyama Otubo ${ }^{1}$, José Augusto Cardoso Filho1, Rodolpho Gareti Carreira1, Bárbarah Brancaleone Coradin².
\end{abstract}

\section{RESUMO}

Objetivo: Relatar um caso de Pneumonia necrotizante e comparar sua conduta com a presente na literatura. Relato do caso: Paciente pediátrico, do sexo feminino, 1 ano e 7 meses, referenciada de serviço de saúde secundário duma cidade de pequeno porte do interior do Estado de São Paulo para o nosso serviço, com quadro de tosse seca, febre e dispneia. Ao exame físico, apresentava redução de murmúrio vesicular associada a submacicez em base de pulmão esquerdo e hipertimpanismo em ápice ipsilateral. Na tomografia de tórax foi evidenciado presença de pneumotórax e imagens sugestivas de cavitações. Foi então submetida a procedimento de ressecção em cunha de um terço do lobo inferior esquerdo e recebeu antibioticoterapia com Ceftriaxona e Oxacilina. Os mesmos demonstraram falha terapêutica, com necessidade de abordagem cirúrgica. Evoluiu com melhora nos padrões laboratoriais e de imagem sendo mantida antibioticoterapia por 21 dias. Considerações finais: A pneumonia adquirida na comunidade (PAC) tem elevada prevalência na população pediátrica e pode evoluir com variadas complicações, dentre elas a pneumonia necrotizante, caracterizada por formações necróticas no tecido pulmonar devido a um processo infeccioso. A pneumonia necrotizante pode evoluir com complicações graves para o paciente, sendo necessária eficiência no manejo e tratamento dessa enfermidade.

Palavras-chave: Pneumonia, Complicações, Antibiótico.

\begin{abstract}
Objective: To report a case of necrotizing pneumonia and compare its conduct with that found in literature. Case report: Pediatric female patient, 1 year and 7 months, referred from a secondary health service of a small city in the interior of São Paulo for our service with dry cough, fever and dyspnea. Upon physical examination, the patient presented with reduced vesicular murmur associated with left lung-based submacicez and ipsilateral apex hyperpanism. Chest tomography showed the presence of pneumothorax and images suggestive of cavitations. Patient was then submitted to resection of one third of the left lower lobe and received antibiotic therapy with Ceftriaxone and Oxacillin. Therapeutic failure took place, requiring a surgical approach. The patient evolved with improvement in laboratory and imaging results and antibiotic therapy was maintained for 21 days. Final considerations: Community-acquired pneumonia (CAP) has a high prevalence in the pediatric population and may evolve with various complications, including necrotizing pneumonia, characterized by necrotic formations in lung tissue due to an infectious process. Necrotizing pneumonia can evolve with severe complications for the patient, requiring efficiency in the management and treatment of the disease.
\end{abstract}

Keywords: Pneumonia, Complications, Antibiotic.

1 Universidade de Franca, Franca-SP. *E-mal: matheusgoncalvessousa@hotmail.com

${ }^{2}$ Santa Casa de Franca, Franca-SP. 


\section{RESUMEN}

Objetivo: Informar un caso de neumonía necrotizante y comparar su conducta con encontrada en la literatura. Caso clínico: paciente pediátrica, 1 año y 7 meses, derivada de un servicio de salud secundario de una pequeña ciudad en el interior de São Paulo para nuestro servicio con tos seca, fiebre y disnea. En el examen físico, el paciente presentó soplo vesicular reducido asociado con submacicez izquierdo basado en el pulmón e hiperpanismo del ápice ipsilateral. Tomografía de tórax mostró la presencia de neumotórax e imágenes sugestivas de cavitación. Luego fue sometida a un procedimiento de resección en cuña de un tercio del lóbulo inferior izquierdo y recibió terapia con antibióticos con ceftriaxona y oxacilina. Demostraron fracaso terapéutico, requiriendo abordaje quirúrgico. Evolucionado una mejora en los estándares de laboratorio y de imagen, la terapia con antibióticos se mantuvo durante 21 días. Consideraciones finales: Neumonía adquirida en la comunidad (NAC) tiene una alta prevalencia en la población pediátrica y puede evolucionar con diversas complicaciones, incluida la neumonía necrotizante, caracterizada por formaciones necróticas en el tejido pulmonar debido a un proceso infeccioso. La neumonía necrotizante puede evolucionar con complicaciones graves para el paciente, que requiere un manejo y tratamiento eficientes de esta enfermedad.

Palabras clave: Neumonía, Complicaciones, Antibiótico.

\section{INTRODUÇÃO}

A pneumonia adquirida na Comunidade (PAC) consiste numa das causas de óbito mais relevantes na população pediátrica em países em desenvolvimento. A incidência é estimada em aproximadamente 0,29 episódio/ano, em número de casos, corresponde 150 milhões de infecções anuais. No ano de 2017, o número absoluto de falecimentos, devido à PAC, na população pediátrica, no Brasil, foi cerca de 1151 casos, segundo dados do DATASUS. Os principais agentes etiológicos, dependerá da faixa etária do paciente.

Nas crianças, no primeiro ano de vida, 90\% dos microrganismos correspondem a vírus, com maior destaque para o Vírus Sincicial Respiratório, e outros com menor prevalência como influenza, parainfluenza, adenovírus, rinovírus. Posteriormente predomina os quadros bacterianos. Sendo o principal agente o Streptococcus pneumoniae, e um menor número de casos relacionado com Stafilococcus aureus, Haemophilus influenzae, etc. (Diretrizes brasileiras em PAC em Pediatria, 2007; Departamento Científico de Pneumologia, et al., 2018).

No seu diagnóstico, um reconhecimento detalhado dos sintomas como tosse, febre, aumento de incursões respiratórias, sinal de sofrimento respiratórios (uso de musculatura acessória, principalmente a subcostal), queda da oxigenação e dor torácica, são de importante valia, uma vez que os achados laboratoriais podem ser inespecíficos e a radiografia de tórax, possui indicações particulares como dúvida diagnóstica, pneumonia com sinais de gravidade, fracasso terapêutico, e pacientes hospitalizados, então sua realização não é recomendado de maneira rotineira (Departamento Científico de Pneumologia, et al., 2018).

Os casos não complicados podem ser tratados ambulatorialmente, os critérios para internação desses pacientes são: idade menor que 2 meses, quadro sugestivo de sepse, hipoxemia com necessidade de oxigênio complementar, insuficiência respiratória aguda, impossibilidade de administrar medicamentos por via oral, condições sociais que não propiciem reavaliação, comorbidades que influenciem o curso clínico e presença de complicações (RODRIGUES JC, et al., 2016; HARRIS M, et al., 2011).

$\mathrm{Na}$ ausência desses critérios, o tratamento é feito de forma ambulatorial, com 7 dias de amoxicilina e reavaliação em 48 horas ou caso ocorra piora clínica. Nos casos graves pode-se administrar ampicilina ou penicilina cristalina e quando idade inferior a 2 meses associar gentamicina para cobertura contra bactérias gram-negativas. Na suspeita de Pneumonias atípicas os macrolídeos são os mais indicados (Departamento Científico de Pneumologia, et al., 2018; VERVLOET LA, et al., 2007).

Quando não melhora clínica do paciente em 72 horas com uso de antibiótico, deve-se considerar cepas bacterianas resistência ou surgimento de alguma complicação, dentre as principais: abcesso pulmonar, derrame pleural e pneumatocele. Uma complicação rara, mas de alta gravidade é a Pneumonia Necrotizante 
caracterizada pelo aparecimento de microcavitações numa área de consolidação (SOCIEDADE BRASILEIRA DE PNEUMOLOGIA E TISIOLOGIA, et al., 2011).

Este trabalho tem como objetivo descrever um caso clínico de pneumonia necrotizante, uma complicação rara da pneumonia adquirida na comunidade em paciente pediatrico, assim como abordar sua conduta e terapeutica utilizados.

\section{DETALHAMENTO DO CASO}

Paciente do sexo feminino, 1 ano e 7 meses, parda, foi encaminha de um serviço secundário de saúde localizado numa cidade de pequeno porte do interior do Estado de São Paulo para o nosso departamento com quadro de tosse seca, febre e dispneia. Segundo relato da mãe, primeiramente surgiu um quadro febril há cerca de 8 dias, aferida em domicilio, com única medida no valor de $39^{\circ} \mathrm{C}$, associado a rinorreia não purulenta e tosse seca. Foi levada a Unidade de Pronto Atendimento (UPA), onde foi medicada com amoxicilina e dipirona, pois foi levantada a hipótese de rinossinusite bacteriana, com tratamento domiciliar.

Apresentou melhora temporária do quadro e dois dias depois apresentou novo pico febril com piora do estado geral e prostração, procurou novamente a UPA, mas dessa vez foi encaminhada ao hospital regional da mesma cidade, local que permaneceu internada durante cinco dias, sem melhora do seu quadro. Não havia fatores de risco para a doença, nem exposição ao tabagismo passivo. Nos antecedentes pessoais não havia relato de comorbidades ou histórico de pneumopatias prévias.

Não fazia uso de medicamentos contínuos. Nos antecedentes familiares, mãe negou que parentes de primeiro grau possuam alguma patologia relevante para o caso. Ao exame físico, de entrada, encontrava-se em regular estado geral, ativa e reativa. Sinais vitais: Frequência cardíaca 144 batimentos por minuto, Frequência respiratória 42 incursões respiratórias, Saturação venosa periférica 100\%; Glicemia capilar: 96 $\mathrm{mg} / \mathrm{dL}$, afebril (temperatura axilar de $35^{\circ} \mathrm{C}$ ).

No aparelho cardiovascular 2 bulhas normofonéticas em 2 tempos, sem sopros. Na avaliação torácica, expansibilidade pulmonar simétrica bilateral, sem lesões externas, com som claro pulmonar, enfisema subcutâneo da base direita até o abdome, murmúrio veicular sem ruídos adventícios. No exame abdominal, nada relevante para o caso. Após a admissão, foi realizado procedimento de decorticação pulmonar e encaminhada à Unidade de Terapia intensiva, local que efetuaram extubação sem intercorrências, e colocada em assistência ventilatória com cateter de oxigênio.

Nos exames laboratoriais da admissão, destacava-se, Hemograma sem alterações na série vermelha, no leucograma, mostra leucocitose (23700) com neutrofilia (Segmentados 57\% e Bastonetes 13\%), linfopenia (linfócitos típicos $25 \%$ e atípicos $0 \%$ ) e presença de granulações tóxicas em $60 \%$ dos neutrófilos, além de plaquetose (805.000).

Marcadores de atividade inflamatória (Proteína C Reativa: 134). Gasometria sem alterações. Realizado radiografia de tórax para verificar posicionamento do cateter venoso Central. Controle da drenagem pleural à esquerda e enfisema de partes moles.

Foram levantadas hipóteses de PAC com derrame pleural, e pós-operatório de decorticação pulmonar. Foram prescritos ceftriaxona $600 \mathrm{mg}$, de $12 \mathrm{em} 12$ horas e Oxacilina $600 \mathrm{mg}$, de 6 em 6 horas. Mantida dieta em jejum. Solução em Holliday-Segar 100\%, e medicamentos analgésicos e antipiréticos. Após 1 dia em UTI, foi encaminhada para enfermaria pediátrica para monitorização do quadro. As hemoculturas não permitiram a identificação do agente etiológico. O resultado do anatomopatológico da decorticação apontou, na microscopia: pleurite exsudativa inespecífica, sem sinais de especificidade ou malignidade na amostra.

Paciente evoluiu com piora dos sintomas álgicos, embora tenha tido melhora dos parâmetros laboratoriais, como marcador de atividade inflamatória (Proteina $C$ reativa: 54,3 ) e melhora do leucograma (índices da serie branca dentre dos valores de referência para a idade). Devido a não melhora dos parâmetros clínicos, foi feita nova tomografia computadorizada de tórax, evidenciando sinais sugestivos de cavitações, permitindo assim a hipótese de pneumonia necrotizante. No Quarto dia de internação, já que não ocorria melhora clínica com 
antibioticoterapia, paciente foi submetida a ressecção em cunha de um terço do lobo inferior do pulmão esquerdo e biopsia a céu aberto.

Depois do procedimento paciente progrediu com melhora dos parâmetros clínicos, com drenagem de aproximados $100 \mathrm{~mL}$ de conteúdo serossanguinolento no $2^{0}$ dia pós-operatório. Foi realizado nova Tomografia Computadorizada de tórax, o qual demonstrou controle da drenagem de coleção pleural à esquerda, enfisema de partes moles, colabamento parcial do pulmão esquerdo, com sinais de espessamento da pleural visceral e parietal.

Foi mantida antibioticoterapia com oxacilina até completar ciclo de 21 dias, evolução da dieta para recomendada para a idade, foi agendado remoção do Dreno após finalização do antibiótico e reavaliação para analisar condições de alta da paciente.

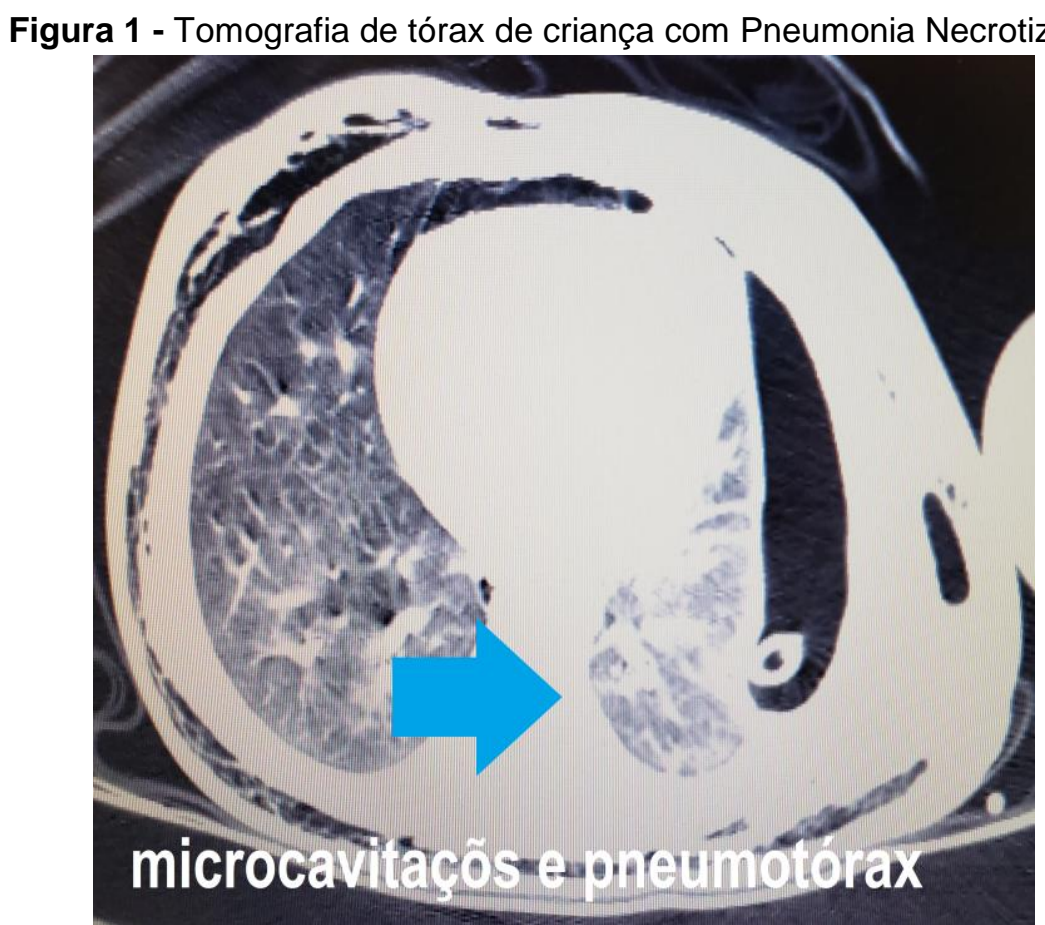

Fonte: Sousa MG, et al., 2020

\section{DISCUSSÃO}

A pneumonia necrotizante (PNM) pode ser explicada como uma complicação da PAC, na qual sucedesse um processo de desvitalização do epitélio respiratório e posteriormente evolução para focos necróticos periféricos numa área de consolidação (WHESTPHAL FL, et al., 2009).

O mecanismo fisiopatológico da PNM ainda não está devidamente elucidado, mas sabe-se que a infecção por um microrganismo, associado com algumas especificidades dos hospedeiros, dentre elas pode-se citar, estados de imunossupressão, como estilismo, tabagismo, idade avançada, diabetes mellitus, doença pulmonar crônica, doença hepática, todos esses fatores, possibilitam para acarretar num processo inflamatório exacerbado e localizado, com uma insuficiência dos mecanismos contrarregulatórios.

Como consequência, acontecerá a congestão da vascularização brônquica, com formação de microtrombos e obstrução vascular. A isquemia cursa com necrose liquefativa tecidual e formação de múltiplas e pequenas cavidades (SCHANDERT L, et al., 2009; NORTE A, et al., 2012).

Dentre os agentes etiológicos causadores da PNM, o de importância mais significativa é o Streptococcus pneumoniae, outros de menor incidência que também estão envolvidos na infecção: Streptococus virians, Haemophilus influenza, Klebsiella pneumoniae, dentre outros. Um agente que merece referência é o 
Staphylococcus aureus resistente à meticilina, associado com quadro muito grave de PNM necrotizante, antes sua infecção era restrita ao ambiente hospitalar e em grupos de risco, pacientes idosos, uso prolongado de corticoides, acesso venoso central, mas atualmente, devido ao surgimento de novas cepas, já existe vários relatos na literatura de contágio no ambiente comunitário e em pessoas imunocompetentes (SCHANDERT L, et al., 2009; Lorber B, 2005).

O diagnóstico apoia-se no conjunto entre quadro clínico e achados laboratoriais e de imagem. As manifestações desses pacientes variam entre um curso insidioso, com queixas inespecíficas, a um início abrupto que evolui com insuficiência respiratória franca.

A tomografia computadorizada mostra-se um importante recurso diagnóstico, demonstrando locais de hipodensidade e numerosas microcavitações, que podem coexistir com níveis hidroaéreos. A radiografia de tórax, método muito usado, pode-se visualizar colapso pulmonar ou presença de fístula bronco-pleural, mas o exame é insatisfatório para um correto estudo das condições pulmonares na PNM (NORTE A, et al., 2012; SCHANDERT L, et al., 2009; Tzeng DZ, et al., 2007).

A análise laboratorial nesses pacientes pode revelar, alterações no hemograma, como modificação quantitativa de leucócitos, geralmente com neutrofilia, outros achados como queda no valor de hemoglobina e de plaquetas, que ocorre em casos graves e em patologias crônicas. A pesquisa do agente etiológico é de extrema relevância para o direcionamento terapêutico, as amostras incorporam pesquisa do escarro e hemocultura. Mas o início do tratamento deve ser feito, de maneira empírica, para evitar a expansão do processo infeccioso (SCHANDERT L, et al., 2009).

As possibilidades terapêuticas na PMN, levantam opiniões bastante divergentes. Uma linha de autores defende uma abordagem mais conservadora, no sentido de instituir de forma muito precoce antibioticoterapia, com cobertura ampla para anaeróbios (penicilina de largo espectro ou cefalosporina de $3^{\underline{a}}$ ou $4^{\underline{a}}$ geração).

Sendo a abordagem cirúrgica resguardada para os casos com maior gravidade, dentre essas complicações pode-se destacar: fístulas bronco-pleurais de alto débito, insuficiência respiratória, sepse e empiema com encarceramento pulmonar (WESTPHAL FL, et al., 2010; NORTE A, et al., 2012).

Uma outra linha, adora uma postura mais agressiva, advogando que, logo após a investigação apontar a presença de uma área necrótica, o segmento ou lobo comprometido deve ser removido cirurgicamente. Nesse caso, a abordagem cirúrgica, deve ser imediata, devido à possibilidade dessa área comprometida se estender a mais de um segmento. Nessa situação, é necessário pneumectomia (WESTPHAL FL, et al., 2010; AYED AK, et al., 2005).

O desfecho cirúrgico está estritamente relacionado com a dimensão da área necrótica e estado patológico do paciente. Vastas regiões que sofreram liquefação, estão com maiores índices de mortalidade, quando equiparado com quadros focais. Algumas complicações são passivas de ocorrer, devido ao processo cirúrgico, os principais são: fístulas broncopleurais prolongadas, necessidade de ventilação mecânica, e cavidades residuais, neste caso, exigindo reintervenção cirúrgica.

No pós-operatório, um cuidado ativo e rápido do setor de fisioterapia é de suma importância para reabilitações pulmonar e motora desses pacientes (WESTPHAL FL, et al., 2010; PARRA MN, et al., 2016; Tsai YF, et al., 2011)

No caso clínico abordado, a paciente possuía uma situação clínica compatível com o quadro de PNM, primeiramente pelas manifestações sintomáticas apresentadas pela mesma, com rápida evolução para insuficiência respiratória franca, precedido por sintomas de tosse, taquipneia com uso de musculatura acessória, e picos febris. Na parte laboratorial, aumento de Proteína $\mathrm{C}$ reativa e leucocitose, com necessidade de internação pelos critérios de hipoxemia e insuficiência respiratória

Após alguns dias de evolução, com deteriorização clínica, o manejo de antibióticos empiricamente mostrou-se ineficaz, foi optado por repetição da Tomografia, o achado de derrame pleural e colabamento parcial de pulmão esquerdo, e microcavitações em área de condensação, principal característica radiológica de PNM, conforme citado por Schandert L, et al., 2009. Tudo isso contribuiu para corroborar a hipótese diagnóstica. Mesmo com terapia com antibióticos de amplo espectro de amplo espectro, com oxacilina, que 
corresponde a penicilina resistente as betalactamases, e ceftriaxona (cefalosporina de $3^{a}$ geração) para cobertura dos principais agentes etiológicos, esquema semelhante trazido em Westphal FL, et al., 2010, a paciente continuou com piora das condições clínicas, então após longo debate com o setor de cirurgia pediátrica e repetição do exame tomográfico, foi decido pela abordagem cirúrgica, com remoção do lobo pulmonar comprometido.

A pneumonia necrotizante consiste numa complicação da PAC que, embora tenha baixa incidência, é uma patologia de extrema relevância, pois exige um manejo clínico ou cirúrgico, dependendo do protocolo de cada serviço, extremamente antecipada nesses pacientes, pelo fato de envolver microrganismos de elevada patogenicidade e risco de progressão para complicações mais severas.

\section{REFERÊNCIAS}

1. BEDRAN RM, et al. Pneumonias adquiridas na comunidade na infância e adolescência. Revista Médica de Minas Gerais, 2012; 22(7): S40-S47.

2. Departamento Científico de Pneumologia, Sociedade Brasileira de Pediatria. Pneumonia adquirida na comunidade na infância. Sociedade Brasileira de Pediatria, 2018; 3:1-7.

3. DIRETRIZES BRASILEIRAS EM PAC EM PEDIATRIA. Diretrizes brasileiras em pneumonia adquirida na comunidade em pediatría - 2007. Jornal Brasileiro de Pneumologia, 2007; 33(1): S31-S50.

4. NORTE A, et al. Pneumonia necrotizante: uma complicação rara. Revista Científica da Ordem dos Médicos 2012; 25(1):51-55.

5. PARRA NM, et al. Neumonías necrosantes graves con empiema asociado. Revista Chilena de Cirugía, 2016; 68(5):379-383.

6. RODRIGUES JC, SILVA FILHO LVRF. Pneumonias agudas na criança. Boletim da Sociedade de Pediatria de São Paulo, 2016; 1(5):4-7.

7. SCHANDERT L, et al. Pneumonia necrotizante: Relato de caso. Revista Brasileira de Clínica Médica, $2009 ; 7: 21$-23.

8. SOCIEDADE BRASILEIRA DE PNEUMOLOGIA E TISIOLOGIA, et al. Pneumonia adquirida na comunidade na infância: tratamento e prevenção. Diretrizes clínicas na saúde suplementar, 2011; 3-16.

9. WESTPHAL FL, et al. Tratamento cirúrgico de crianças com pneumonia necrosante. Jornal Brasileiro de Pneumologia, 2010; 36(6):716-723.

10. AYED AK, AL-ROWAYEH A. Lung resection in children for infectious pulmonary diseases. Pediatr Surg Int. 2005;21(8):604-8.

11. TSAI YF, et al. Surgical treatment of 26 patients with necrotizing pneumonia. Eur Surg Res. 2011; 47:13-8.

12. LORBER B. Lung Abscess, In: Mandell GL, Bennet JD, Dolin R. Principles and Practice of Infectious Diseases. 6th Ed, Philadelphia: Elsevier Churchille Livingstone, 2005;853-856.

13. Tzeng DZ, Markman M, Hardin K. Necrotizing Pneumonia and Pulmonary Gangrene - Difficulty in Diagnosis, Evaluation and Treatment. Clin Pulmonary Med 2007;14(3):166-170

14. HARRIS M, et al. BTS guidelines for the management of community acquired pneumonia in children: update 2011. Thorax. 2011;66 Suppl 2: ii-1-23.

15. VERVLOET LA, et al. Infection by Mycoplasma pneumoniae and its importance as an etiological agent in childhood community-acquired pneumonias. Braz J Infec Dis. 2007;11(5):507-14 